\title{
B-cell maturation antigen expression across hematologic cancers: a systematic literature review
}

\author{
Ahmet Dogan (10)', David Siegel ${ }^{2}$, Nguyet $\operatorname{Tran}^{3}$, Alan Fu', Jessica Fowler ${ }^{3}$, Rajesh Belani ${ }^{3}$ and Ola Landgren (1)
}

\begin{abstract}
B-cell maturation antigen (BCMA) plays a critical role in regulating B-cell proliferation and survival. There is evidence for BCMA expression in various hematologic malignancies, suggesting that BCMA may play an important role as a biomarker or therapeutic target in these diseases. Given advances in understanding the role of BCMA in B-cell development and the promise of BCMA as a therapeutic target, a systematic review is needed to rigorously assess the evidence for BCMA expression and identify areas of consensus and future research. The objective of this review was to summarize the evidence on BCMA protein and mRNA expression across hematologic malignancies. Using a PubMed database search up to 28 August 2019, a systematic literature review of publications reporting BCMA expression in patients with hematologic malignancies was conducted. Data from published congress abstracts presented at the American Society of Clinical Oncology and the American Society of Hematology were also searched. Studies that assessed BCMA expression (protein or mRNA) in patients of any age with hematologic malignancies were included. A total of 21 studies met inclusion criteria and were included in the review. BCMA was expressed in several hematologic malignancies, including multiple myeloma (MM), chronic lymphocytic leukemia, acute B-lymphoblastic leukemia, non-Hodgkin lymphoma (NHL), and Hodgkin lymphoma. BCMA was expressed at uniformly high levels across all $13 \mathrm{MM}$ studies and at low to moderate levels in acute myeloid leukemia and acute lymphoblastic leukemia. These results suggest that BCMA is a relevant target in MM as well as in a subset of B-cell leukemia. BCMA expression in Hodgkin lymphoma and NHL varied across studies, and further research is needed to determine the utility of BCMA as an antibody target and biomarker in these diseases. Differences in sample type, timing of sample collection, and laboratory technique used may have affected the reporting of BCMA levels.
\end{abstract}

\section{Introduction}

B-cell maturation antigen (BCMA) plays a critical role in regulating $\mathrm{B}$-cell proliferation and survival, as well as differentiation into plasma cells ${ }^{1,2}$. BCMA is shed from the surface of plasma cells via $\gamma$-secretase-mediated cleavage, resulting in a soluble form $(\mathrm{sBCMA})^{3}$. The effects of BCMA expression have been studied extensively in multiple myeloma (MM), and BCMA can be targeted to achieve anti-tumor effects in MM patients ${ }^{4}$.

\footnotetext{
Correspondence: Ahmet Dogan (dogana@mskcc.org)

${ }^{1}$ Memorial Sloan Kettering Cancer Center, New York, NY, USA

${ }^{2} J$ ohn Theurer Cancer Center at Hackensack University Medical Center, Hackensack, NJ, USA

Full list of author information is available at the end of the article
}

Clinical trials of anti-BCMA therapies, including antibody-drug conjugates, BiTE ${ }^{\circledR}$ (Bispecific T-cell engager) molecules, and chimeric antigen receptor $\mathrm{T}$ (CAR T) cell therapy, have demonstrated promising efficacy $^{5-9}$. There is also evidence of BCMA expression in other hematologic malignancies, including B-cell leukemias and lymphomas ${ }^{10,11}$.

There is currently a lack of systematic reviews integrating knowledge of BCMA expression in hematologic malignancies. The primary objective of this review is to systematically assess the scientific literature and identify which hematologic cancers express BCMA. This review will summarize the evidence for BCMA expression levels in patients with hematologic malignancies. 


\section{Methods}

Data collection and study identification

This review was fully compliant with the 2009 preferred reporting items for systematic reviews and Meta-Analysis guidelines for the reporting of systematic reviews ${ }^{12}$. A systematic literature review of English-language publications reporting BCMA expression in patients with hematologic malignancies was conducted using a PubMed database search up to 28 August 2019. Data from published congress abstracts presented at the American Society of Clinical Oncology and the American Society of Hematology were also searched. Studies that reported on patients of any age with hematologic malignancies and assessed BCMA expression (protein or mRNA) were included. Broad search terms, including terms for MM, leukemia, lymphoma, and BCMA expression, were used to capture potentially relevant literature. The full list of search terms is shown in Table 1. Reference lists of included publications, as well as reference lists of other systematic reviews, were also searched for relevant publications. Animal studies, studies using non-human and/or non-hematologic cell lines, studies investigating non-hematologic conditions, review articles, comments, editorials, letters, guidelines, legal cases, debates, newspaper articles, opinions, protocols, workshops, patient education brochures, and non-English publications were excluded.

Table 1 Publication database search strategies.

\begin{tabular}{ll}
\hline Pub Med search terms & Filters \\
\hline "Multiple myeloma" OR "Acute lymphoblastic & Species: Humans \\
leukemia" OR "Acute myelogenous leukemia" OR & \\
"Chronic lymphocytic leukemia" OR "Chronic & \\
myelogenous leukemia" OR "Acute monocytic & \\
leukemia" OR "Hodgkin's lymphoma" OR "Nodular & \\
sclerosing HL" OR "Mixed-cellularity subtype HL" OR \\
"Diffuse large B-cell lymphoma" OR "Anaplastic large \\
cell lymphoma" OR "Burkitt lymphoma" OR \\
"Lymphoblastic lymphoma" OR "Mantle cell \\
lymphoma" OR "Peripheral T-cell lymphoma" OR \\
"Transformed follicular and transformed mucosa- \\
associated lymphoid tissue lymphoma" OR "Follicular \\
lymphoma" OR "Cutaneous T-cell lymphoma" OR \\
"mycosis fungoides" OR "Sézary syndrome" OR \\
"Lymphoplasmacytic lymphoma Waldenström \\
macroglobulinemia" OR "Marginal zone B-cell \\
lymphoma" OR "MALT lymphoma" OR "Small cell \\
lymphocytic lymphoma" OR "chronic lymphocytic \\
leukemia") AND ("BCMA expression" OR "BCMA" OR \\
"B-Cell Maturation Antigen" OR "TNFRSF17" OR \\
"TNFRSF17 expression" \\
\hline
\end{tabular}

\section{Study selection}

Two independent reviewers from Amgen, Inc. first screened the title and abstracts returned from the search criteria and, in the event of ambiguous content relevance, proceeded with an in-depth review of the main text. Any disagreements between the reviewers with respect to content relevance was resolved by a third reviewer from Amgen, Inc.

\section{Data abstraction}

Data including country or region, sample size, patient characteristics, hematologic malignancy studied, tumor burden, sample type, method of cell preparation/purification, laboratory technique, cutoff for BCMA positivity, level of BCMA expression (mRNA or protein) investigated, and percentage of patients with BCMA expression were extracted from studies included in this review. When data from congress abstracts were also in full-text publications, only unique data were extracted.

\section{Results}

Using the aforementioned criteria and search terms, the search yielded 616 studies. After excluding animal studies, 428 studies were reviewed, of which 21 met inclusion criteria; 3 studies were identified from reference lists of review publications (Fig. 1). Study information and methodology, patient characteristics, and percentage of patients with BCMA expression are summarized in Table 2 and described below. BCMA protein and mRNA expression across hematologic malignancies is summarized in Table 3, with additional details presented in the text. All studies were conducted in the United States unless otherwise specified.

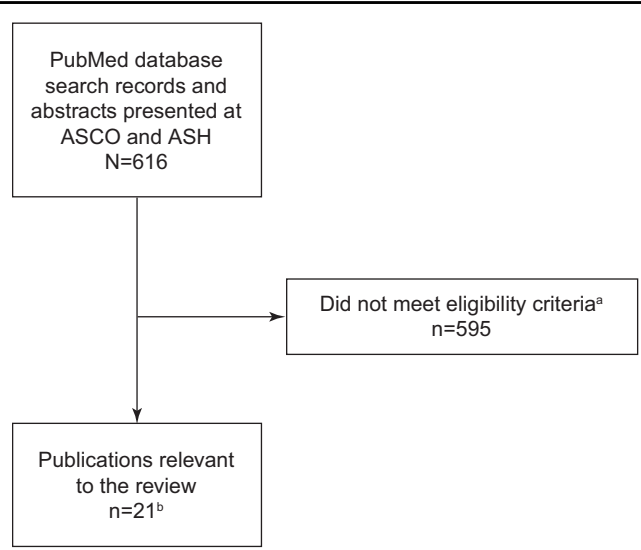

Fig. 1 Identification of relevant publications. a Animal studies, studies using non-human and/or non-hematologic cell lines, studies investigating non-hematologic conditions, review articles, comments, editorials, letters, guidelines, legal cases, debates, newspaper articles, opinions, protocols, workshops, patient education brochures, and non-English publications were excluded. b Three studies were identified from reference lists of review publications. 


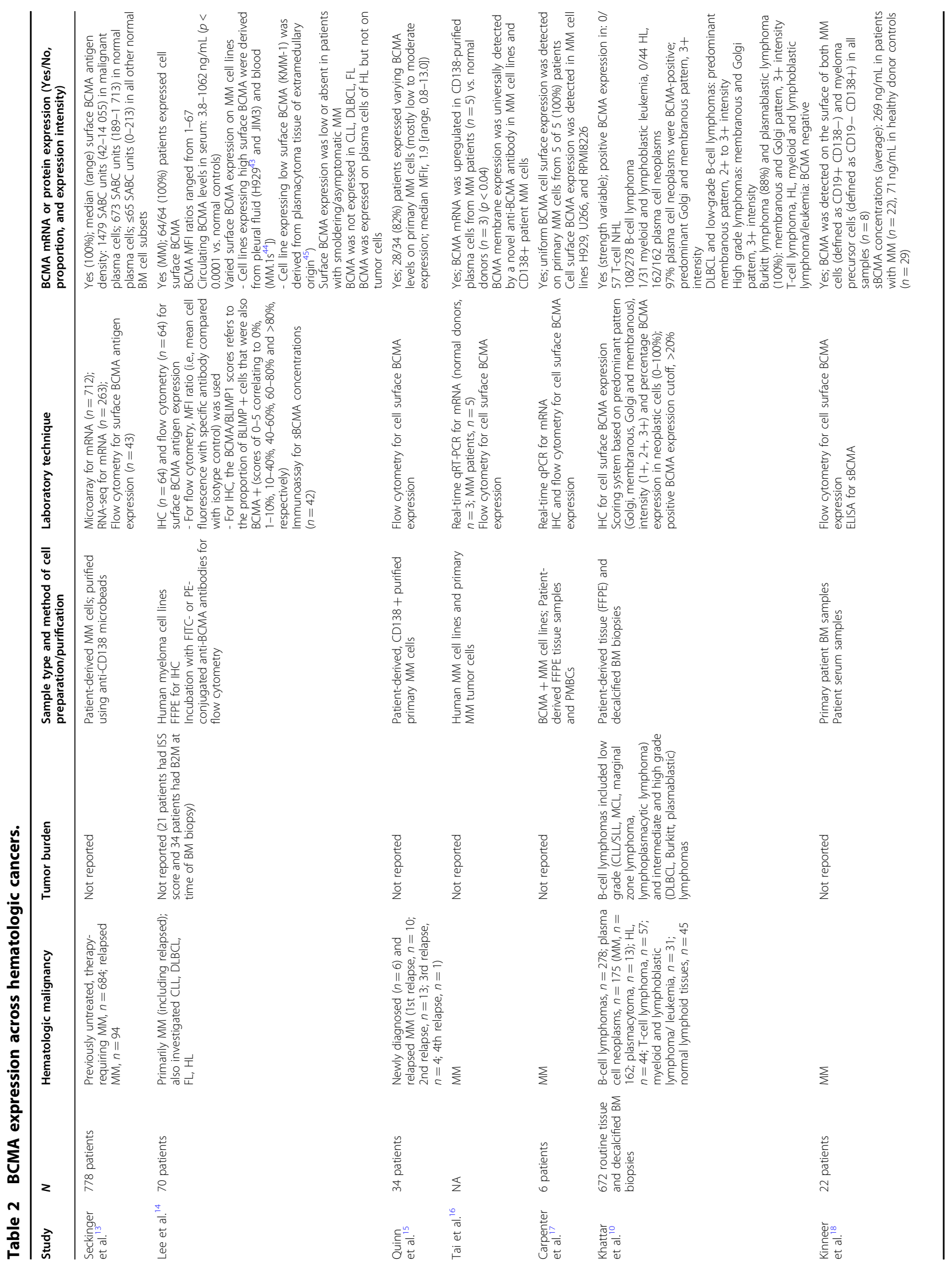




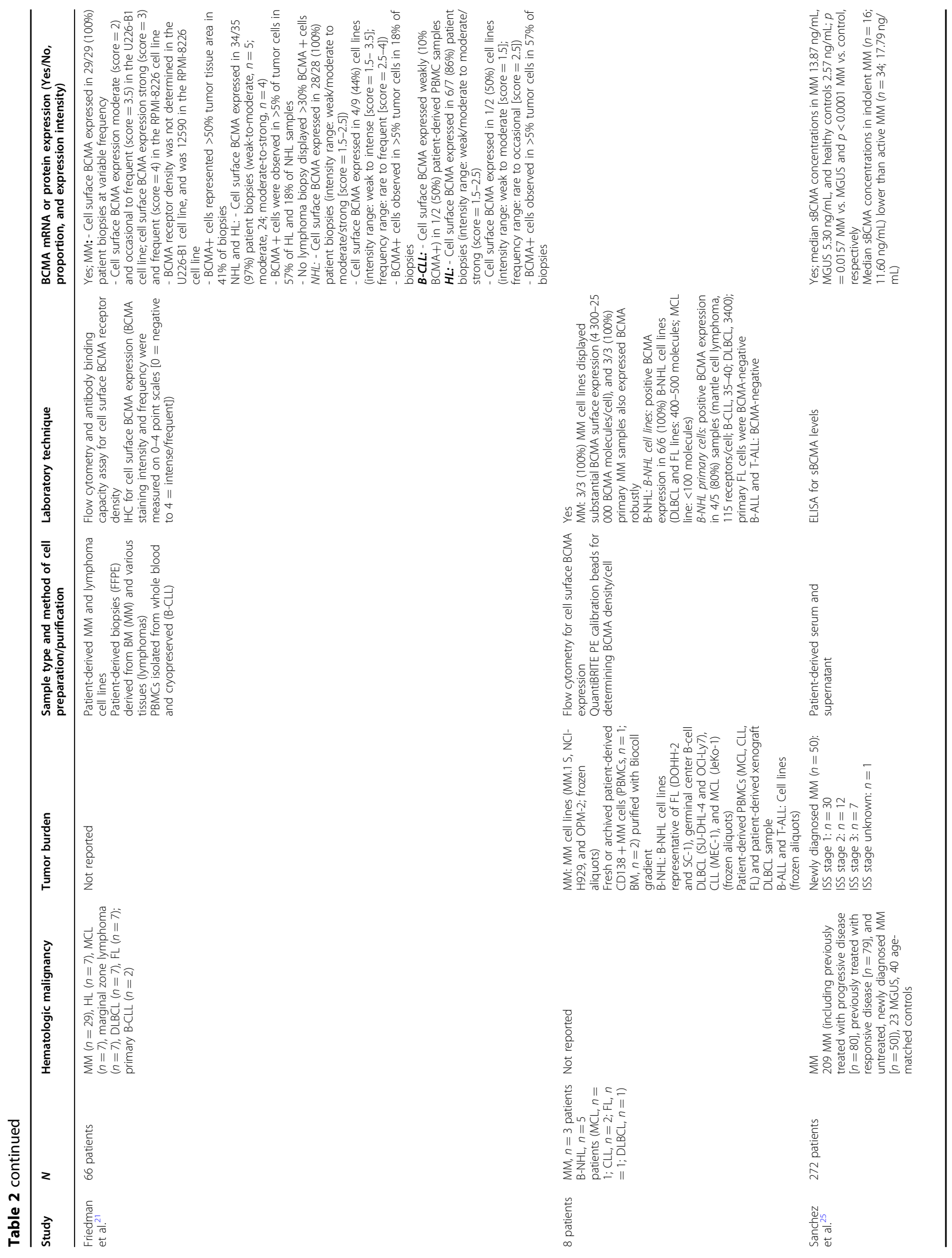




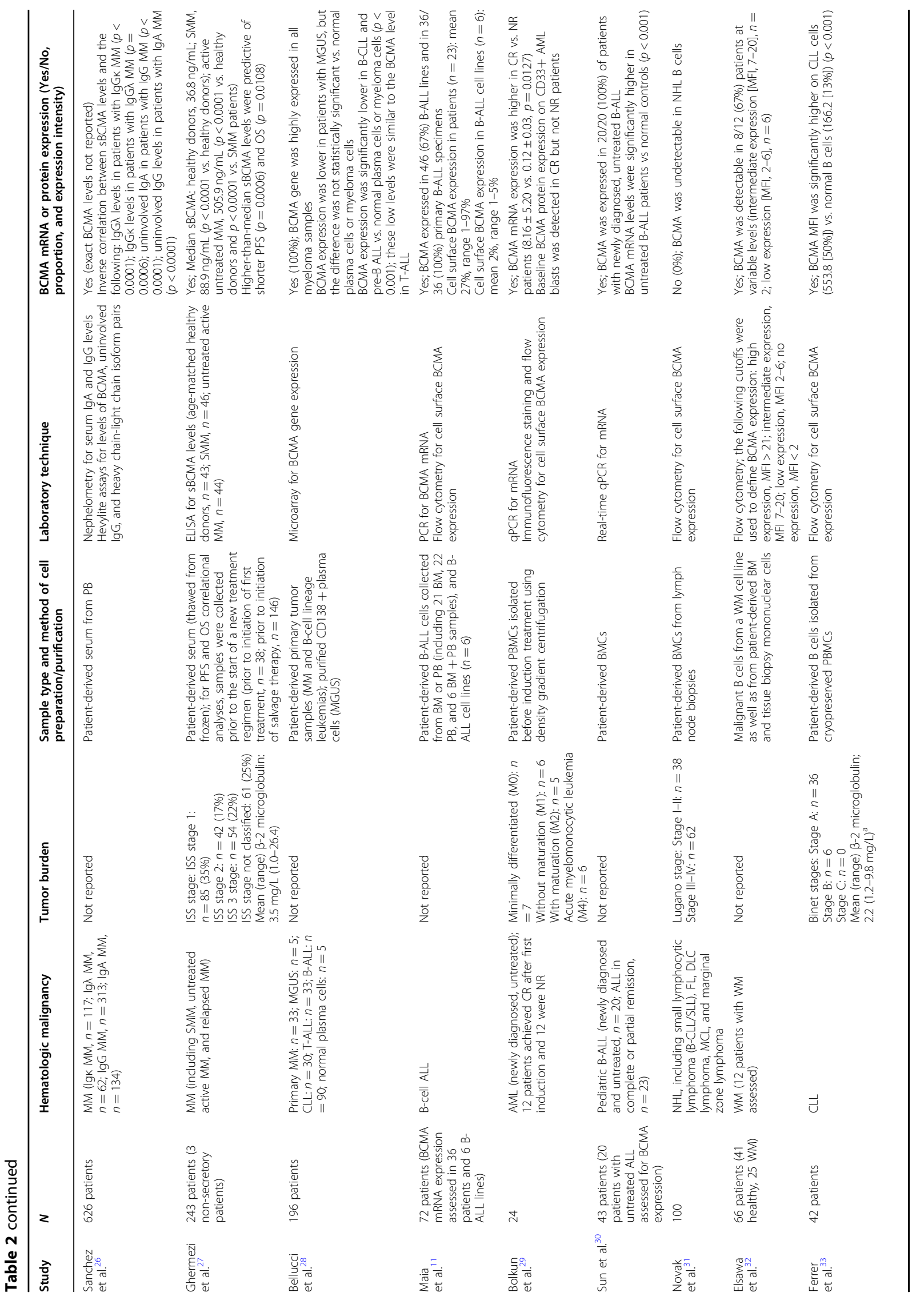




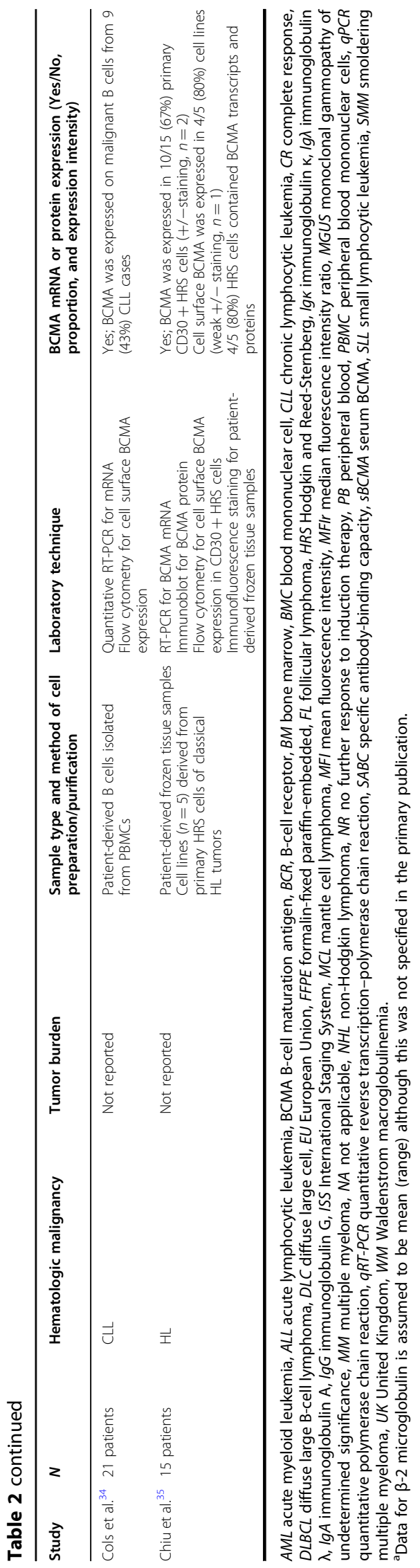

\section{BCMA expression in MM}

Of the 13 studies evaluating BCMA expression in MM, all reported detectable BCMA expression.

\section{Cell surface and intracellular BCMA protein expression}

In a European study by Seckinger et al. ${ }^{13}$, BCMA was identified as a potential therapeutic target in newly diagnosed MM (NDMM) or relapsed MM. Cell surface BCMA expression was measured as specific antibodybinding capacity $(\mathrm{SABC})$ units using multidimensional flow cytometry. Samples from 31 previously untreated MM patients and 12 patients with relapsed MM were analyzed. Surface BCMA was expressed on malignant plasma cells of previously untreated and relapsed patients with MM (median of 1479 SABC units; range, 42-14,055). The expression was higher on the malignant cells compared with both normal plasma cells (median of 673 SABC units; range, 189-173) and other bone marrow (BM) cells subsets (median $\leq 65$ SABC units; range $0-213$ ). Surface BCMA expression on plasma cells (normal or malignant) was significantly higher $(p<0.001)$ than that of the normal, non-plasma cells.

A United Kingdom (UK) study of 70 patients with MM found that BCMA expression is maintained through relapse, extramedullary spread, and residual disease following therapy ${ }^{14}$. BCMA expression was assessed in patient-derived $\mathrm{BM}$ aspirate MM cells using flow cytometry $(n=64)$ and immunohistochemistry (IHC; $n=64$ ). All (64/64) patients with symptomatic MM expressed cell surface and intracellular BCMA at varying levels by IHC. The median BCMA mean fluorescence intensity (MFI) ratio was 4.1 (range, 1-67) as measured by flow cytometry. Higher levels of cell surface BCMA were associated with shorter progression-free survival (PFS) and overall survival (OS) in patients with both high- or standard-risk cytogenetics, indicating that cell surface BCMA levels may have independent prognostic value when used with conventional cytogenetic assessments. Cell surface BCMA expression was also assessed in human MM cell lines by flow cytometry, and results are summarized in Table 2.

In a UK study of patients with NDMM $(n=6)$ and relapsed $(n=28)$ MM, cell surface BCMA levels were analyzed in primary MM cells from the BM of patients who were off therapy ${ }^{15}$. All 28 evaluable patients expressed BCMA; levels generally ranged from low to moderate. The median fluorescence intensity ratio was 1.9 (range, 0.8-13.0), as analyzed by flow cytometry.

Tai et al. ${ }^{16}$ found that BCMA is universally expressed on the $M M$ cell surface and its expression is restricted to plasma cells. Cell surface BCMA protein expression was measured by MFI using flow cytometry analysis. BCMA membrane expression was universally detected by an antiBCMA antibody in MM cell lines and CD138+ patientderived MM cells $(n=12)$. Carpenter et al. ${ }^{17}$ assessed 
Table 3 Reference guide for BCMA protein and mRNA expression across hematologic malignancies.

\begin{tabular}{|c|c|c|c|}
\hline Tumor type & $\begin{array}{l}\text { Cell } \\
\text { surface BCMA }\end{array}$ & sBCMA & BCMA mRNA \\
\hline Multiple myeloma & $+^{10,13,14,18-23}$ & $++^{14,21,24-26}$ & $++^{13,19,20,27}$ \\
\hline T-ALL & -23 & NA & $++^{a, 27}$ \\
\hline B-ALL & -23 & NA & $+^{11,27,29}$ \\
\hline Mature B-cell neoplasms & $\begin{array}{l}+{ }^{10,22} \\
-{ }^{30}\end{array}$ & NA & NA \\
\hline B-cell lymphoma & $+^{10}$ & NA & NA \\
\hline DLBCL & $\begin{array}{l}-{ }^{14,30} \\
+^{10,22,23}\end{array}$ & NA & NA \\
\hline Plasmablastic lymphoma & $++^{10}$ & NA & NA \\
\hline Burkitt's lymphoma & $+{ }^{10,22}$ & NA & \\
\hline CLL or CLL/SLL & $\begin{array}{l}-{ }^{14,30} \\
+{ }^{22,23,32,33}\end{array}$ & NA & $+^{a, 27,33}$ \\
\hline $\mathrm{FL}$ & $\begin{array}{l}-{ }^{14,30} \\
+{ }^{10,22,23}\end{array}$ & NA & NA \\
\hline $\mathrm{MCL}$ & $\begin{array}{l}-{ }^{30} \\
+22,23\end{array}$ & NA & NA \\
\hline Marginal zone lymphoma & $\begin{array}{l}-30 \\
+22\end{array}$ & NA & NA \\
\hline $\begin{array}{l}\text { Waldenstrom's } \\
\text { macroglobulinemia }\end{array}$ & $+^{31}$ & NA & NA \\
\hline $\mathrm{HL}^{\mathrm{b}}$ & $\begin{array}{l}-10,14 \\
+^{22,34}\end{array}$ & NA & $++^{34}$ \\
\hline T-cell lymphoma & $-{ }^{10}$ & NA & NA \\
\hline $\begin{array}{l}\text { Myeloid and lymphoblastic } \\
\text { Iymphoma/leukemia }\end{array}$ & $-{ }^{10}$ & NA & NA \\
\hline $\mathrm{AML}^{\mathrm{c}}$ & $++^{28}$ & NA & $+^{28}$ \\
\hline
\end{tabular}

+ positive BCMA expression, - negative BCMA expression, $A M L$ acute myeloid leukemia, $B$-ALL B-cell acute lymphoblastic leukemia, BCMA B-cell maturation antigen, $C L L$ chronic lymphocytic leukemia, $D L B C L$ diffuse large B-cell lymphoma, HL Hodgkin lymphoma, FL follicular lymphoma, $M C L$ mantle cell lymphoma, NA not applicable, $N H L$ non-Hodgkin lymphoma, $S B C M A$ serum BCMA, SLL small lymphocytic leukemia, T-ALL T-cell acute lymphocytic leukemia, WM Waldenstrom's macroglobulinemia.

${ }^{a}$ Very low expression reported in Bellucci et al. ${ }^{28}$.

${ }^{b}$ In Lee ${ }^{14}$, BCMA was expressed on plasma cells of $\mathrm{HL}$ but not on tumor cells. cln Bolkun et al. ${ }^{29}$, BCMA protein expression on CD33 + AML blasts was detected in patients who experienced complete remission after first induction, but not in non-responders.

expression in BCMA-positive MM cell lines, formalinfixed paraffin-embedded (FFPE) tissue samples, and peripheral blood mononuclear cells (PBMCs) derived from six patients with MM. Cell surface BCMA was measured as the number of events using flow cytometry, and IHC staining was performed with BCMA and isotype-matched control antibodies on consecutive sections. Using flow cytometry analysis, BCMA expression was detected on $\mathrm{MM}$ cell lines but not on other types of cell lines, including primary CD34+ hematopoietic cells. BCMA was uniformly expressed on the surface of primary MM cells all patients $(n=5)$, as determined by flow cytometry or IHC.

Khattar et al. ${ }^{10}$ evaluated cell surface and intracellular BCMA expression in 672 biopsies from normal lymphoid tissues and from patients with various hematologic malignancies, including MM, using IHC staining. FFPE tissue and decalcified BM biopsies were collected from patients with plasma cell neoplasms, including normal lymphoid tissues $(n=45)$, MM $(n=162)$, and plasmacytoma $(n=13)$. B-cell lineage malignancies and plasma cell neoplasms had high BCMA expression levels. All MM samples and $97 \%$ of plasma cell neoplasms were BCMApositive and had a membranous and Golgi staining pattern with strong $(3+)$ intensity.

Kinneer et al. ${ }^{18}$ analyzed BCMA expression on the surface of MM cells and myeloma progenitor cells (MPCs) in primary BM samples $(n=8)$ of MM patients. Unlike mature MM cells, MPCs lack a mature plasma cell phenotype and are not fully differentiated ${ }^{19}$. However, MPCs can transfer the disease and predict a poorer patient response to stem cell transplant and proteasome inhibitor treatment ${ }^{20}$, making them an important cell population to characterize. Using flow cytometry, histograms were generated on the MPCs and the MM plasma cell population. BCMA was detected on the cell surface of both MM cells and MPCs of all samples analyzed.

Friedman et al. ${ }^{21}$ evaluated cell surface BCMA expression in $2 \mathrm{MM}$ cell lines derived from peripheral blood (PB) $\left(\mathrm{U} 266-\mathrm{B}^{22}, \mathrm{RPMI}-8226^{23}\right)$ and in $29 \mathrm{MM} \mathrm{BM}$ biopsies. BCMA expression in cell lines is summarized in Table 2. For BM samples from patients with MM, IHC demonstrated that 29/29 (100\%) samples expressed BCMA, although the percentage of sample staining positive was variable. BCMA-positive cells represented over half of the tumor tissue area in $41 \%$ of the biopsies.

In a German study by Bluhm et al. ${ }^{24}$, cell surface BCMA expression was assessed in $3 \mathrm{MM}$ cell lines and in primary, patient-derived PBMCs $(n=1$ patient) and BMderived cells ( $n=2$ patients) using flow cytometry. The MM cell lines displayed substantial BCMA surface expression (mean: 4300-25,000 BCMA molecules/cell), and primary MM cells also expressed BCMA robustly.

\section{sBCMA levels}

In a study of 209 patients with MM, sBCMA levels in NDMM patients $(n=50$; median $13.87 \mathrm{ng} / \mathrm{mL})$ were significantly higher than in age- and gender-matched healthy controls $(n=40$; median $2.57 \mathrm{ng} / \mathrm{mL} ; p<0.0001)$ and in patients with monoclonal gammopathy of undetermined significance (MGUS; $n=23 ; \quad$ median $5.30 \mathrm{ng} / \mathrm{mL}$; $p=0.0157)^{25}$. Patients with indolent MM $(n=16)$ had 
lower sBCMA levels (median $11.60 \mathrm{ng} / \mathrm{mL}$ ) than those with active MM $(n=34$; median $17.79 \mathrm{ng} / \mathrm{mL})$. In addition, BCMA levels in patients with MM correlated with clinical response and OS. Responsive ( $\geq$ partial response [PR]) patients ( $n=80$ samples) had lower sBCMA levels than patients $(n=79$ samples $)$ with progressive disease (median $4.06 \mathrm{ng} / \mathrm{mL}$ vs. $19.76 \mathrm{ng} / \mathrm{mL}$, respectively; $p=$ $0.0038)$. OS was significantly shorter in MM patients $(n=162)$ who had BCMA levels above the median concentration $(10.85 \mathrm{ng} / \mathrm{mL})$ vs. those with levels below the median $(p=0.0014)$. A subsequent study by Sanchez et al. $^{26}$ showed that sBCMA levels inversely correlated with uninvolved polyclonal antibody production in patients with MM, suggesting a possible sBCMAmediated mechanism of immune deficiency in these patients. In addition, BCMA-BAFF complexes were detected in PB-derived sera of patients with MM $(n=12$ total), as determined by ELISA.

Further building upon the Sanchez et al. studies ${ }^{25,26}$, Ghermezi et al. ${ }^{27}$ identified sBCMA as a biomarker that can monitor and predict outcomes for MM patients. Serum was collected from 243 patients with MM and sBCMA levels were determined by ELISA. sBCMA levels were higher among patients with smoldering MM (SMM; $n=46 ; 88.9 \mathrm{ng} / \mathrm{mL}[p<0.0001$ vs. healthy donors $])$ and untreated active MM $(n=44 ; 505.9 \mathrm{ng} / \mathrm{mL}[p<0.0001$ vs. healthy donors and $p<0.0001$ vs. SMM patients]) than in age-matched healthy donors $(n=43$; median sBCMA of $36.8 \mathrm{ng} / \mathrm{mL}$ ). In addition, sBCMA levels correlated with the proportion of plasma cells in BM biopsies (Spearman's rho $=0.710 ; p<0.001$ ), clinical status (complete response [CR] vs. PR, $p=0.0374$; CR vs. progressive disease, $p<$ 0.0001), and M-protein levels. Furthermore, higher-thanmedian sBCMA levels were predictive of a shorter PFS $(p=0.0006)$ and OS $(p=0.0108)$. sBCMA levels also correlated with changes in M-protein and serum-free light chain levels in individual patients with MM.

In the study by Lee et al. ${ }^{14}$, sBCMA in patient samples $(n=42)$ ranged from $3.8-1062 \mathrm{ng} / \mathrm{mL}(p<0.0001$ vs. normal controls), with no difference between NDMM patients vs. those with relapsed disease. In contrast to the reports by Sanchez et al. ${ }^{25}$ and Ghermezi et al $^{25}$, there was no association between sBCMA level and treatment response or survival.

The study by Kinneer et al. ${ }^{18}$ measured sBCMA concentrations in serum samples from patients with MM and age- and sex-matched healthy donors. As determined by ELISA, sBCMA levels were higher in patients with MM (mean sBCMA: $269 \mathrm{ng} / \mathrm{mL}, n=22$ ) compared with healthy donor controls (average $71 \mathrm{ng} / \mathrm{mL}, n=27$ ).

\section{BCMA mRNA expression}

In the study by Seckinger et al. ${ }^{13}$, BCMA mRNA expression was assessed using gene expression profiling by
DNA microarray $(n=712)$ and RNA sequencing $(n=263)$ in patients with NDMM or relapsed/refractory MM. BCMA mRNA was expressed in all patient samples, supporting the observed increase in cell surface BCMA expression by flow cytometry. The study by Tai et al. ${ }^{16}$ used real-time quantitative reverse-transcription-polymerase chain reaction (RT-qPCR) to quantify BCMA mRNA. BCMA mRNA was upregulated in CD138-purified plasma cells from patients with MM $(n=5)$ vs. normal donors $(n=3)(p<0.04)$. These findings supported the enhanced cell surface BCMA protein expression observed by flow cytometry. Plasmacytoid dendritic cells had detectable BCMA mRNA at significantly lower levels than CD138+ plasma cells ( $p<0.005$ for each paired sample) from either patients with MM or normal donors. BCMA mRNA was increased in plasmacytoid dendritic cells from patients with MM vs. normal donors $(p<0.03)$.

In the study by Carpenter et al. ${ }^{17}, \mathrm{BCMA}$ had a restricted expression pattern, as assessed by qPCR. The BCMA expression of a plasmacytoma sample from a patient with advanced MM ( 3500 BCMA cDNA copies/ $10^{5}$ cDNA actin copies) was dramatically higher than the BCMA expression of cDNA samples from several types of normal tissues (all $<500$ BCMA cDNA copies $/ 10^{5}$ cDNA actin copies). These results were in line with previously described findings of increased cell surface BCMA protein, as assessed by IHC and flow cytometry.

Bellucci et al. ${ }^{28}$ characterized BCMA expression in primary tumor samples from MM patients $(n=33)$ and in purified CD138+ plasma cells from MGUS patients $(n=5)$ and normal plasma cells $(n=5)$ using high-density oligonucleotide microarrays. BCMA was highly expressed in all MM samples. BCMA expression was lower in patients with MGUS, but the difference was not statistically significant compared with normal plasma cells or MM cells.

\section{BCMA expression in acute leukemia}

Of the five studies evaluating BCMA expression in leukemia, 4/5 (80\%) reported BCMA expression. BCMA expression was assessed in two studies of acute Blymphoblastic leukemia (B-ALL), two studies that investigated both T-ALL and B-ALL, and one acute myeloid leukemia (AML) study.

\section{Cell surface BCMA protein expression}

In a study by Maia et al. ${ }^{11}(n=72)$, cell surface BCMA was assessed in primary B-ALL cells and B-ALL cell lines. B-ALL cells were collected from patients' $\mathrm{BM}$ or $\mathrm{PB}$, and included $21 \mathrm{BM}, 22 \mathrm{~PB}$, and $6 \mathrm{BM}$ plus $\mathrm{PB}$ samples. Flow cytometry was used to assess BCMA cell surface expression in primary B-ALL $(n=23)$ and B-ALL lines $(n=6)$. Mean surface BCMA expression (percentage of cells) was $27 \%$ in patients and $2 \%$ in B-ALL cell lines. 
In the study by Bluhm et al. ${ }^{24}$, BCMA cell surface expression was examined in 2 B-ALL cell lines (NALM6 and $\mathrm{REH}$ ). Both cell lines were BCMA-negative, as assessed by flow cytometry.

In a Polish study of 24 patients with newly diagnosed AML, cell surface BCMA protein expression was assessed in patient-derived $\mathrm{PBMCs}^{29}$. Patient AML ranged from minimally differentiated to acute myelomonocytic leukemia. Twelve patients achieved complete remission (CR) after first induction and 12 were non-responders (NR). Immunofluorescence staining showed that baseline BCMA protein expression on CD33 + AML blasts was present in patients who achieved CR $(16 \% \pm 9 \%$ of cells) but not in patients classified as NR ( $<1 \%$ of cells).

In the study by Bluhm et al. ${ }^{24}$, BCMA was not expressed in the T-ALL cell line Jurkat, as assessed by flow cytometry.

\section{BCMA mRNA expression}

In a Chinese study by Sun et al. ${ }^{30}$, plasma samples were collected from 43 pediatric patients with B-ALL. Among the enrolled patients, 20 had newly diagnosed B-ALL and 23 patients were in complete or partial remission after previous treatment. Using real-time qPCR, BCMA mRNA expression was assessed in BMCs from 20 newly diagnosed B-ALL patients and 15 normal controls. BCMA was expressed in $20(100 \%)$ patients with newly diagnosed BALL and was found to be significantly higher in BMCs from patients with B-ALL compared with controls $(p<0.001)$.

In the study by Maia et al. ${ }^{11}$, BCMA mRNA expression was assessed in primary B-ALL cells (BM or PB; $n=36$ ) and B-ALL lines $(n=6)$, using RT-PCR. BCMA was detected in 36/36 (100\%) primary B-ALL specimens and in 4/6 (67\%) B-ALL lines. In the study by Bolkun et al. ${ }^{29}$, BCMA mRNA expression was assessed in patient-derived PBMCs from 24 patients with newly diagnosed $\mathrm{AML}^{29}$. BCMA mRNA expression was higher in CR vs. NR patients $(8.16 \pm 5.20$ vs. $0.12 \pm 0.03, p=0.0127)$, as determined by qPCR.

In the study by Bellucci et al. ${ }^{28}$, BCMA expression was characterized in primary tumor samples from patients with T-ALL $(n=33)$ and B-ALL $(n=90)$ using highdensity oligonucleotide microarrays ${ }^{28}$. BCMA expression was significantly lower in B-ALL compared with normal plasma cells or MM cells $(p<0.001)$; these low levels were similar to the BCMA level in T-ALL.

\section{$B C M A$ expression in mature $B$-cell neoplasms}

Of the 10 studies evaluating BCMA expression in lymphoma, six studies assessed patients with nonHodgkin lymphoma $(\mathrm{NHL})^{24,28,31-34}$, one study assessed patients with Hodgkin lymphoma $(\mathrm{HL})^{35}$, and three studies assessed a mixed NHL and HL population ${ }^{10,14,21}$. Among these studies, 7/9 (78\%) reported BCMA expression in NHL and 2/4 (50\%) reported BCMA expression in HL.

\section{Cell surface BCMA protein expression}

In the study by Khattar et al. ${ }^{10}$, FFPE tissue and decalcified BM biopsies were collected from patients with Bcell lymphomas $(n=278)$. T-cell lymphoma $(n=57)$, myeloid and lymphoblastic lymphoma/leukemia $(n=31)$, HL $(n=44)$ and normal lymphoid tissue $(n=45)$ samples were also collected. Diffuse large B-cell lymphoma (DLBCL) and low-grade B-cell lymphoma samples predominantly showed a membranous pattern with $2+$ to $3+$ staining intensity using IHC. In DLBCL, 39\% of patients with the active B-cell subtype and $35 \%$ of patients with the germinal center B-cell subtype had $>20 \%$ BCMA expression in neoplastic cells. There were no significant differences in the intensity and pattern of staining. Eight cases (88\%) of Burkitt lymphoma and seven cases (100\%) of plasmablastic lymphoma showed membranous and Golgi BCMA staining patterns with $3+$ intensity. BCMA was expressed in only $1 / 31$ (3\%) samples of myeloid and lymphoblastic lymphoma/leukemia and was not expressed in any samples of T-cell lymphoma or HL.

In a study of 100 patients with NHL, cell surface BCMA expression was analyzed in BMCs from lymph node biopsies using flow cytometry ${ }^{31}$. NHL samples were diagnosed with either small lymphocytic lymphoma (BCLL/SLL), follicular lymphoma (FL), DLBCL, MCL, or marginal zone lymphoma. In contrast to the study by Khattar et al. ${ }^{10}$, which identified intermediate to high BCMA expression in several types of NHL, BCMA was undetectable in NHL B cells.

Elsawa et al. ${ }^{32}$ analyzed cell surface BCMA expression in malignant $B$ cells from a Waldenstrom's macroglobulinemia cell line and from patient-derived BM and tissue biopsy mononuclear cells. BCMA was detectable at variable levels via flow cytometry in 8/12 (67\%) patients, with $2 / 12(17 \%)$ patients having intermediate expression and 6/12 (50\%) patients having low expression.

Ferrer et al. ${ }^{33}$ investigated cell surface BCMA protein expression in 42 patients with CLL who had available cryopreserved PBMC samples at the time of diagnosis. By flow cytometry, BCMA MFI was significantly higher on CLL cells (553.8, 50\% of positive cells for BCMA on CD19 + cells) than on normal B cells $(166.2,13 \%$ of positive cells for BCMA on CD19+ cells) $(p<0.001)$. BCMA expression correlated with unmutated $I G H V$ genes and poor-risk cytogenetics but was not correlated with disease stage, blood lymphocyte count, ZAP70, or CD38 expression. In addition, patients with a higher BCMA expression on CLL cells had a shorter PFS compared with patients with lower BCMA expression (median, 57 months vs. 206 months; $p=0.021$ ). In a study of 21 patients with CLL, cell surface BCMA was expressed on malignant $B$ 
cells from 9/21 (43\%) CLL cases, as assessed by flow cytometry $^{34}$.

In the study by Friedman et al. ${ }^{21}$, cell surface BCMA expression was evaluated in 9 NHL cell lines (myelogenous leukemia, $n=2$; ALL, $n=2$; MCL, $n=2$; Burkitt's lymphoma, $n=2$; B-CLL, $n=1$ ) using IHC. BCMA was expressed in 4/9 (44\%) cell lines. Of these, BCMA staining ranged from weak to intense (score $=1.5-3.5)$ and rare to frequent (score $=2.5-4$ ). Cell surface BCMA expression was also assessed in 28 biopsies from patients with NHL ( $n=7$ each for DLBCL, MCL, FL, and mantle zone lymphoma) using IHC. BCMA was expressed in all NHL biopsies, with staining intensity ranging from weak/ moderate to moderate/strong (score $=1.5-2.5$ ). In addition, BCMA-positive cells were observed in $>5 \%$ of the tumor cells in $18 \%$ of NHL patient biopsies. Cell surface BCMA expression was also evaluated in $2 \mathrm{HL}$ cell lines, of which 1/2 (50\%) expressed BCMA at weak to moderate intensity $($ score $=1.5$ ) and rare to occasional frequency (score $=2.5$ ). BCMA was expressed in 6/7 (86\%) HL biopsies, with staining intensity ranging from weak/ moderate to moderate/strong (score $=1.5-2.5$ ). In addition, BCMA + cells were observed in $>5 \%$ of the tumor cells in 57\% of HL biopsies. Furthermore, 1/2 (50\%) patient-derived PBMC samples was shown to be weakly (10\%) BCMA+ by flow cytometry.

The study by Bluhm et al. ${ }^{24}$ measured cell surface BCMA expression in B-NHL cell lines representative of FL, germinal center B-cell DLBCL, CLL, and MCL. BCMA was expressed in all 6 B-NHL cell lines. The BCMA frequencies in the DLBCL and FL cell lines ranged from 400-500 molecules, and the MCL cell line had a BCMA frequency of $<100$ molecules. Primary B-NHL cells (CD19 + CD20 + CD138-) from patients with MCL $(n=1)$, CLL $(n=2)$, and FL $(n=1)$, as well as a patientderived xenograft DLBCL sample, were also assessed for BCMA expression. Patient samples were derived from PBMCs, and residual CD138+ cells were excluded from analysis. BCMA was expressed on the following primary B-NHL samples: MCL, 115 receptors/cell; B-CLL, 35-40 receptors/cell; DLBCL, 3400 receptors/cell. Primary FL cells were BCMA-negative.

Chiu et al. ${ }^{35}$ evaluated cell surface BCMA expression in patients with HL. Cell lines $(n=5)$ were derived from primary Hodgkin and Reed-Sternberg (HRS) cells of classical HL tumors. Cell surface BCMA was expressed at variable levels in $4 / 5(80 \%)$ cell lines, as determined by flow cytometry. BCMA protein was also expressed in $4 / 5$ $(80 \%)$ cell lines, as assessed by immunoblot. Frozen tissue samples were collected from 15 patients with classical HL, including subtypes of nodular sclerosis (11), mixed cellularity $(n=3)$, and lymphocyte depletion $(n=1)$. Primary CD30 + HRS cells from classical HL tumors expressed BCMA in 10/15 (67\%) cases examined.

\section{BCMA mRNA expression}

In the study by Cols et al. ${ }^{34}$, BCMA mRNA expression was measured using RT-qPCR in 21 patients with CLL. BCMA transcripts increased after CLL cells were incubated with CD40L, a molecule aberrantly expressed by CLL cells.

In the study by Bellucci et al. ${ }^{28}$, BCMA mRNA expression was characterized in primary tumor samples from patients with (CLL) $(n=30)$. As measured by highdensity oligonucleotide microarrays, BCMA expression was significantly lower in B-CLL compared with normal plasma cells or MM cells $(p<0.001)$.

In the study by Chiu et al. ${ }^{35}$, BCMA transcript and protein were expressed in 4/5 (80\%) HL cell lines, as assessed by RT-PCR ${ }^{35}$. In contrast to the BCMA expression results reported in Chiu et al. ${ }^{35}$, BCMA expression was absent in CLL, DLBCL, and FL, and on classical HL tumor cells, in the study by Lee et al. ${ }^{14}$.

\section{Discussion}

In this systematic review, BCMA expression was found across multiple hematologic malignancies of precursor Bcells, plasma cells or late-stage B cells (Fig. 2). In MM, all identified studies reported detectable BCMA expression in all patient samples via protein or mRNA, often at high levels. These results were consistent despite the variability in the number of included patients, laboratory techniques used to assess BCMA levels, and sample types in each study.

\section{MM}

In the MM studies, cell surface BCMA expression was seen in MGUS, NDMM, or relapsed MM at varying levels. BCMA expression was generally lower in MGUS compared with $\mathrm{MM}^{25,28}$. Three studies reported higher sBCMA levels in patients with NDMM compared with controls $^{14,25,27}$, with one of these studies finding that sBCMA levels were similar between NDMM and relapsed $\mathrm{MM}^{14}$.

Based on these studies, the treatment status of MM generally does not appear to have a significant influence on BCMA protein or BCMA mRNA expression. BCMA protein and mRNA expression was reported in both NDMM and relapsed MM with expression maintained in residual disease and at relapse. Patients with myeloma precursor disease (e.g., MGUS) did not have statistically significant differences in BCMA cellular expression compared with patients with $\mathrm{MM}^{28}$, but an increase in sBCMA correlated with more active/advanced disease stage. Most studies either combined NDMM and relapsed MM patient data or did not evaluate BCMA expression by $\mathrm{MM}$ treatment status; therefore, further work is needed to assess BCMA protein (cell surface and sBCMA) and mRNA expression specifically in NDMM or relapsed MM. 


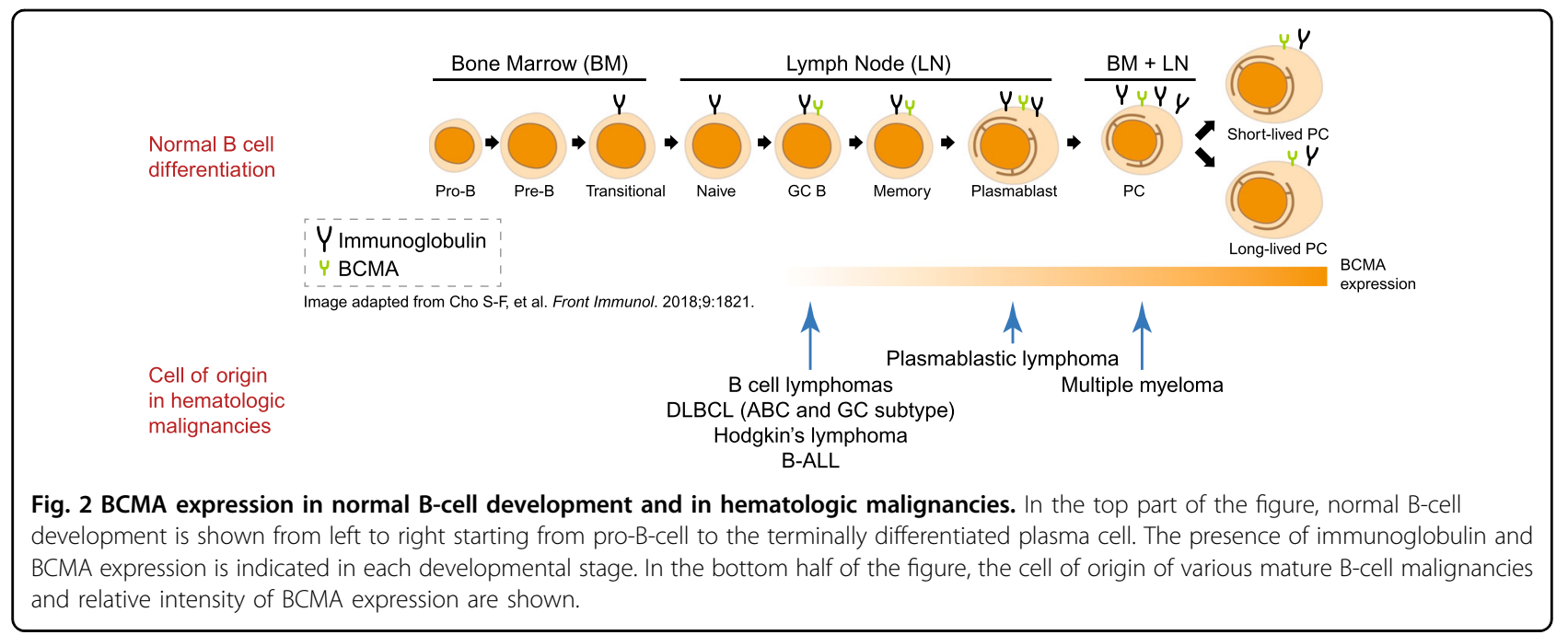

Several studies demonstrated that sBCMA is elevated in $\mathrm{MM}$ and can be used as a biomarker to predict disease response and survival ${ }^{25,27}$. Furthermore, sBCMA can serve as an independent prognostic marker of clinical outcomes $^{27}$. In our analysis, four studies reported higher sBCMA levels in patients with MM compared with healthy controls ${ }^{14,18,25,27}$. Of these, two studies found an association between elevated SBCMA and poor clinical outcomes ${ }^{25,27}$. Ghermezi et al. ${ }^{27}$ found that sBCMA levels were markedly lower in patients with $\mathrm{CR}$ than in those with $<\mathrm{CR}$, and both PFS and OS were shorter among patients whose sBCMA levels were above the median. Similarly, in a study by Sanchez et al, responsive $(\geq P R)$ patients had lower sBCMA levels than patients showing progressive disease and OS was shorter among patients with sBCMA levels above the median ${ }^{25}$. In addition, a study by Sanchez et al demonstrated an inverse correlation between sBCMA levels and levels of uninvolved polyclonal antibodies, suggesting that higher sBCMA levels can be associated with immunoparesis ${ }^{26}$. This evidence supports the role of BCMA as a biomarker and immuno-oncology therapy target in MM.

\section{Acute leukemia}

For leukemia, 4/5 (80\%) identified studies reported BCMA expression at varying levels ${ }^{11,24,28-30}$. B-ALL samples were generally BCMA-positive, with $100 \%$ of patientderived BMCs and B-ALL cells expressing BCMA mRNA in two studies ${ }^{11,30}$. Based on these findings, BCMA may be a promising biomarker of response and immuno-oncology therapy target in B-ALL, but further research is warranted to determine whether anti-BCMA therapies can be effective in these malignancies. Analyses of databases (e.g., The Cancer Genome Atlas) may be particularly useful to generate additional data related to BCMA expression.

\section{Mature B-cell neoplasms}

BCMA expression in HL and NHL varied widely across studies. In some NHL subtypes, BMCA expression or lack thereof was reported with no conflicting results, whereas BCMA expression data were conflicting in HL studies and in some subtypes of NHL. Despite these variations in BCMA expression in some NHL subtypes across studies, BCMA may still be a suitable target for mature B-NHLs. One study validated BCMA expression in DLBCL, FL, $\mathrm{MCL}$, and $\mathrm{CLL}$, and demonstrated that BCMA CAR $\mathrm{T}$ cells eradicated B-NHL cells in vitro and in vivo through target cell lysis ${ }^{24}$. Only 1 NHL study reported data on patient tumor burden ${ }^{31}$, and there were no NHL or HL studies reporting on patient treatment status. Therefore, future work is needed to determine whether BCMA expression is affected by these factors.

\section{BCMA expression methodology and reporting}

The methodologies used to measure BCMA mRNA or cell surface protein expression may have affected the expression levels reported in each analysis. These differences in methodology could explain the conflicting results reported across some studies, particularly for HL and DLBCL (Table 2).

The majority of studies used flow cytometry and/or IHC to assess cell surface BCMA protein levels ${ }^{14,17,21,29,35}$. Flow cytometry has been shown to be more sensitive than IHC, particularly for specimens with few plasma cells, and can objectively quantify BCMA expression by myeloma cells $^{36}$. An advantage of $\mathrm{IHC}$ is that it does not require fresh tissue. In our analysis, for HL and DLBCL, BCMA expression data were conflicting despite the use of IHC staining on patient-derived FFPE tissue samples across several studies ${ }^{10,14,21}$. Variation in ischemic time before fixation, duration of fixation and fixative formula, 
decalcification method, and sample variability may have affected IHC results ${ }^{37,38}$.

BCMA mRNA levels were analyzed in 8/17 (47\%) of studies using several techniques, predominantly qPCR. Previous studies have shown that the cellular concentrations of proteins correlate with the quantity of their corresponding mRNAs, but the correlation is not strong ${ }^{39,40}$. In one report, the squared Pearson's correlation coefficient for protein and mRNA concentrations across organisms was $\sim 0.4$, suggesting that only $\sim 40 \%$ of the variation in protein expression can be explained by changes at the transcript level ${ }^{40}$. Qualitatively, in our analysis, BCMA protein and mRNA data were in agreement within studies that assessed both forms of BCMA. In 4. MM studies that measured sBCMA levels by immunoassay, sBCMA was detected in all MM patients at higher levels than in healthy controls ${ }^{14,18,25,27}$.

The assessment of factors that constitute BCMA positivity or negativity also varied across studies. Elsawa et al defined cutoffs of MFI $>21,7-20,2-6$, and $<2$ for high, intermediate, low, and no expression, respectively, using flow cytometry in a WM cell line and patient-derived tissue $^{32}$. Khattar et al. ${ }^{10}$ defined a positive BCMA cutoff of $>20 \%$ using IHC in B cell lymphoma patient-derived tissue and $\mathrm{BM}$ biopsies. The remaining studies did not explicitly define BCMA expression cutoffs. Thus, reporting of BCMA expression may vary due to different criteria used to determine BCMA positivity or negativity in each study.

\section{Sample type and timing of collection}

In addition to methodological differences, the sample types used in each analysis may have affected reporting of BCMA expression. The studies included in this review assessed BCMA in cell lines derived from various sources and/or patient-derived samples. As no studies in this analysis performed a head-to-head comparison of patient sample types, it is difficult to determine any potential effects of sample type on BCMA expression. The timepoint at which samples were collected may have also impacted assessment of BCMA expression, although any potential effects are challenging to evaluate as only a few studies reported these data ${ }^{27,29,33}$.

To better understand the utility of BCMA as a biomarker or treatment target, it will be important for future studies to longitudinally evaluate BCMA expression dynamics before and after treatment. For example, the cleaving of cell surface BCMA by $\gamma$-secretase results in shedding of the extracellular domain, leading to circulation of $\mathrm{sBCMA}^{3}$. Sufficient sBCMA accumulation in the BM of MM patients may inhibit BCMA CAR-T cell recognition of tumor cells $^{41}$. Recently, $\gamma$-secretase inhibitors have been found to increase surface BCMA levels in MM cell lines and patient tumor samples, and improve tumor recognition by CAR-T cells in vitro ${ }^{41}$. Furthermore, in a phase 1 study of relapsed/ refractory MM patients ( $n=6$ assessable patients), treatment with the $\gamma$-secretase inhibitor JSMD194 in combination with anti-BCMA CAR T cell therapy led to an ORR of $100 \%$ (5 VGPR, $1 \mathrm{PR})^{42}$.

This study had several limitations. Publication bias may have occurred if relevant ongoing or completed studies have not yet been published. Manual literature searches for BCMA expression studies may have resulted in studies being identified in a non-systematic manner. Most studies were conducted in the US or in Europe, limiting the generalizability of results to other populations. Some studies only reported mRNA data, with no protein correlative data. Based on the method with which BCMA is assessed, it is not clear whether cell surface expression is reflected, although IHC and mRNA appear to be good surrogate markers.

In conclusion, BCMA was expressed in several hematologic malignancies, including MM, CLL, ALL, NHL, and HL. BCMA appears to be a relevant target in MM, given its uniformly positive expression across studies. BCMAtargeted therapy may also be promising in patients with certain types of leukemia or lymphoma, as BCMA was also expressed in these malignancies. Further research is needed to determine the utility of BCMA as a biomarker and antibody target in hematologic malignancies for which evidence was conflicting or only 1 or a few studies were conducted ${ }^{6}$.

\section{Acknowledgements}

Medical writing assistance was provided by Sachi Yim, PhD, and Andrew Gomes, PhD, of BlueMomentum, an Ashfield company, part of UDG Healthcare PLC, and was funded by Amgen, Inc. This systematic literature review was funded by Amgen, Inc.

\section{Author details}

${ }^{1}$ Memorial Sloan Kettering Cancer Center, New York, NY, USA. ${ }^{2} J o h n$ Theurer Cancer Center at Hackensack University Medical Center, Hackensack, NJ, USA. ${ }^{3}$ Amgen, Inc., Thousand Oaks, CA, USA

\section{Author contributions}

All authors participated in the conception and design of the study, analysis and interpretation of data, and development of the manuscript and approval of the final version for submission. N.T. and A.F. participated in data collection/ acquisition of data.

\section{Conflict of interest}

N.T., A.F., J.F., and R.B. are employees of and own stock in Amgen, Inc, developer of 2 BiTE $^{\circledR}$ immunotherapies (AMG 420 and AMG 701) and 1 ADC (AMG 224) targeting BCMA. O.L. received grants/research support from Celgene, Amgen, BMS, and Karyopharm; received grants/research support, honoraria, and other from Janssen; received grants/research support and other for Takeda; received honoraria from Celgene, Amgen, and Adaptive Biotech; and received other from Merck. A.D. received honoraria and consulting or advisory role fees from Roche, Corvus Pharmaceuticals, Physicians' Education Resource, Seattle Genetics, Peerview Institute, Oncology Specialty Group, Pharmacyclics, Celgene, Novartis, Takeda, and EUSAPharma; and research grants from National Cancer Institute and Roche. D.S. received honoraria and consulting or advisory role fees from Celgene, Amgen, Merck, Janssen, BMS, Takeda, and Karyopharm; participated in speakers' bureaus for Celgene, Amgen, Merck, Janssen, BMS, and Takeda; and received research funding from Celgene. 


\section{Publisher's note}

Springer Nature remains neutral with regard to jurisdictional claims in published maps and institutional affiliations.

Supplementary Information accompanies this paper at (https://doi.org/ 10.1038/s41408-020-0337-y).

Received: 23 December 2019 Revised: 21 May 2020 Accepted: 26 May 2020 Published online: 30 June 2020

\section{References}

1. Tai, Y. T. \& Anderson, K. C. Targeting B-cell maturation antigen in multiple myeloma. Immunotherapy 7, 1187-1199 (2015).

2. O'Connor, B. P. et al. BCMA is essential for the survival of long-lived bone marrow plasma cells. J. Exp. Med. 199, 91-98 (2004).

3. Laurent, S. A. et al. $Y$-secretase directly sheds the survival receptor BCMA from plasma cells. Nat. Commun. 6, 7333 (2015).

4. Cho, S. F., Anderson, K. C. \& Tai, Y. T. Targeting B cell maturation antigen (BCMA) in multiple myeloma: potential uses of BCMA-based immunotherapy. Front. Immunol. 9, 1821 (2018).

5. Topp, M. S. et al. Anti-B-cell maturation antigen BiTE molecule AMG 420 induces response in multiple myeloma. J. Clin. Oncol. 38, 775-783 (2020).

6. Shah, N. et al. B-cell maturation antigen (BCMA) in multiple myeloma: rationale for targeting and current therapeutic approaches. Leukemia 34, 985-1005 (2020).

7. Cohen, A. D. et al. B cell maturation antigen-specific CAR T cells are clinically active in multiple myeloma. J. Clin. Invest. 129, 2210-2221 (2019).

8. Brudno, J. N. et al. T cells genetically modified to express an anti-B-cell maturation antigen chimeric antigen receptor cause remissions of poorprognosis relapsed multiple myeloma. J. Clin. Oncol. 36, 2267-2280 (2018).

9. Trudel, S. et al. Targeting B-cell maturation antigen with GSK2857916 antibody-drug conjugate in relapsed or refractory multiple myeloma (BMA117159): a dose escalation and expansion phase 1 trial. Lancet Oncol. 19 1641-1653 (2018). Blood 2017;130:741.

10. Khattar, P. et al. B-cell maturation antigen is exclusively expressed in a wide range of B-cell and plasma cell neoplasm and in a potential therapeutic target for Bcma directed therapies. Blood 130, 2755 (2017).

11. Maia, S. et al. Aberrant expression of functional BAFF-system receptors by malignant B-cell precursors impacts leukemia cell survival. PLoS ONE 6, e20787 (2011).

12. Liberati, A. et al. The PRISMA statement for reporting systematic reviews and meta-analyses of studies that evaluate health care interventions: explanation and elaboration. Ann Intern. Med. 151, W65-W94 (2009).

13. Seckinger, A. et al. Target expression, generation, preclinical activity, and pharmacokinetics of the BCMA-T cell bispecific antibody EM801 for multiple myeloma treatment. Cancer Cell 31, 396-410 (2017).

14. Lee, L. et al. Evaluation of B cell maturation antigen as a target for antibody drug conjugate mediated cytotoxicity in multiple myeloma. Br. J. Haematol. 174, 911-922 (2016).

15. Quinn, J. et al. APRIL promotes cell-cycle progression in primary multiple myeloma cells: influence of D-type cyclin group and translocation status. Blood 117, 890-901 (2011)

16. Tai, Y.-T. et al. Novel anti-B-cell maturation antigen antibody-drug conjugate (GSK2857916) selectively induces killing of multiple myeloma. Blood $\mathbf{1 2 3}$ 3128-3138 (2014).

17. Carpenter, R. O. et al. B-cell maturation antigen is a promising target for adoptive T-cell therapy of multiple myeloma. Clin. Cancer Res. 19, 2048-2060 (2013).

18. Kinneer, $K$. et al. Preclinical assessment of an antibody-PBD conjugate that targets BCMA on multiple myeloma and myeloma progenitor cells. Leukemia 33, 766-771 (2019).

19. Pilarski, L. M. \& Belch, A. R. Clonotypic myeloma cells able to xenograft myeloma to nonobese diabetic severe combined immunodeficient mice copurify with $\mathrm{CD} 34(+)$ hematopoietic progenitors. Clin. Cancer Res. 8, 3198-3204 (2002).

20. Reghunathan, R. et al. Clonogenic multiple myeloma cells have shared stemness signature associated with patient survival. Oncotarget 4, 1230-1240 (2013).

21. Friedman, K. M. et al. Effective targeting of multiple B-cell maturation antigenexpressing hematological malignances by anti-B-cell maturation antigen chimeric antigen receptor T cells. Hum. Gene Ther. 29, 585-601 (2018).
22. Nilsson, K. et al. Established immunoglobulin producing myeloma (lgE) and Iymphoblastoid (lgG) cell lines from IgE myeloma patient. Clin. Exp. Immunol. 7, 447-489. (1970).

23. Matsuoka, Y. et al. Production of free light chains of immunoglobulin by a hematopoietic cell line derived from a patient with multiple myeloma. Proc. Soc. Exp. Biol. Med. 125, 1246-1250 (1967).

24. Bluhm, J. et al. CAR T cells with enhanced sensitivity to B cell maturation antigen for the targeting of B cell non-Hodgkin's lymphoma and multiple myeloma. Mol. Ther. 26, 1906-1920 (2018).

25. Sanchez, E. et al. Serum B-cell maturation antigen is elevated in multiple myeloma and correlates with disease status and survival. Br. J. Haematol. 158 727-738 (2012).

26. Sanchez, E. et al. Soluble B-cell maturation antigen mediates tumorinduced immune deficiency in multiple myeloma. Clin. Cancer Res. 22, 3383-3397 (2016)

27. Ghermezi, M. et al. Serum B-cell maturation antigen: a novel biomarker to predict outcomes for multiple myeloma patients. Haematologica 102, 785-795 (2017)

28. Bellucci, R. et al. Graft-versus-tumor response in patients with multiple myeloma is associated with antibody response to BCMA, a plasma-cell membrane receptor. Blood 105, 3945-3950 (2005).

29. Bolkun, L. et al. Involvement of BAFF and APRIL in resistance to apoptosis of acute myeloid leukemia. J. Cancer 7, 1979-1983 (2016).

30. Sun, B. et al. Raised expression of APRIL in Chinese children with acute lymphoblastic leukemia and its clinical implications. J. Pediatr. Hematol. Oncol. 36, 276-280 (2014)

31. Novak, A. J. et al. Expression of BLyS and ist receptors in B-cell non-Hodgkin lymphoma: correlation with disease activity and patient outcome. Blood 104, 2247-2253 (2004)

32. Elsawa, S. F. et al. B-lymphocyte stimulator (BLyS) stimulates immunoglobulin production and malignant B-cell growth in Waldenström macroglobulinemia. Blood 107, 2882-2888 (2006).

33. Ferrer, $\mathrm{G}$. et al. B cell activation through CD40 and IL4R ligation modulates the response of chronic lymphocytic leukaemia cells to BAFF and APRIL. Br. J. Haematol. 164, 570-578 (2014).

34. Cols, M. et al. Stromal endothelial cells establish a bidirectional crosstalk with chronic lymphocytic leukemia cells through the TNF-related factors BAFF, APRIL and CD40L. J. Immunol. 188, 6071-6083 (2012).

35. Chiu, A. et al. Hodgkin lymphoma cells express $\mathrm{TACl}$ and BCMA receptors and generate survival and proliferation signals in response to BAFF and APRIL. Blood 109, 729-739 (2007).

36. Salem, D. A. et al. Quantification of B-cell maturation antigen, a target for novel chimeric antigen receptor T-cell therapy in myeloma. Leuk. Res. 71, 106-111 (2018).

37. Pekmezci, M. et al. The effect of cold ischemia time and/or formalin fixation on estrogen receptor, progesterone receptor, and human epidermal growth factor receptor-2 results in breast carcinoma. Patholog. Res. Int. 2012, 947041 (2012).

38. Engel, K. B. \& Moore, H. M. Effects of preanalytical variables on the detection of proteins by immunohistochemistry in formalin-fixed, paraffin-embedded tissue. Arch. Pathol. Lab. Med. 135, 537-543 (2011).

39. Edfors, F. et al. Gene-specific correlation of RNA and protein levels in human cells and tissues. Mol. Syst. Biol. 12, 883 (2016).

40. Abreau, De. Sousa R. et al. Global signatures of protein and mRNA expression levels. Global signatures of protein and mRNA expression levels. Mol. Biosyst. 5, 1512-1526 (2009).

41. Pont, M. J. et al. $\gamma$-secretase inhibition increases efficacy of BCMA-specific Chimeric Antigen Receptor $\mathrm{T}$ Cells in multiple myeloma. Blood 134, 1585-1597 (2019).

42. Cowan, A. J. et al. Efficacy and safety of fully human Bcma CAR T cells in combination with a gamma secretase inhibitor to increase Bcma surface expression in patients with relapsed or refractory multiple myeloma. Blood 143(Supplement_1), 204 (2019).

43. Gazdar, A. F. et al. Establishment and characterization of a human plasma cell myeloma culture having a rearranged cellular myc proto-oncogene. Blood 67, 1542-1549 (1986)

44. Greenstein, S. et al. Characterization of the MM.1 human multiple myeloma (MM) cell lines: a model system to elucidate the characteristics, behavior, and signaling of steroid-sensitive and -resistant MM cells. Exp. Hematol. 31, 271-282 (2003).

45. Togawa, A. et al. Establishment and characterization of a human myeloma cell line (KMM-1). Int. J. Cancer 29, 495-500 (1982). 